\title{
Tocilizumab's Efficacy in Severe COVID-19 Patients: Fact or Fiction
}

\author{
Ioannis Andrianopoulos ${ }^{1}$, Dimitrios Kantas ${ }^{1,3}$, Ibrahim A. Bajwa², Essa A. Mohamed ${ }^{3}$ \\ 1.Critical Care and Intensive Care Unit, University Hospital of Ioannina, Ioannina, Greece \\ 2.FU Medical College, Foundation University Islamabad, Islamabad, Punjab, Pakistan \\ 3.Department of Cardiovascular Medicine, Mayo Clinic, Rochester, Minnesota, USA
}

Corresponding author: Dimitrios Kantas, MD, Department of Cardiovascular Medicine, Mayo Clinic 200 First Street SW,

Rochester, Minnesota, USA

Received: September 14, 2021; Accepted: September 22, 2021; Published: September 24, 2021

It has been over a year since the discovery of the first known cases of severe acute respiratory syndrome coronavirus 2 (SARS-CoV-2) in Wuhan, China. The ensuing pandemic has caused over four million deaths, economic recession, and serious long-term consequences in multiple aspects of our society. Over this period, there has been significant progress in understanding the pathogenesis and the course of the disease, which led to significant vaccine-development efforts. However, mortality in severe cases of COVID-19 remains high, and medical professionals treating this specific subset of patients need effective treatment modalities to reduce long-term complications and mortality.

As with all infections, the answer resides in the pathogenesis of the disease of interest. Early in the pandemic, the hypothesis of a "cytokine-storm" syndrome in severe COVID-19 patients was predicated on preliminary data; thus, immunomodulating treatments were deployed (steroids, IL-6 and IL-1 receptor inhibitors, etc.), and a series of trials (randomized and non-randomized) commenced. In severe COVID-19 presentation, steroids proved beneficial in reducing mortality and the need for mechanical ventilation, and until recently, no other drug reduced mortality in critically ill COVID-19 patients.

Most patients infected with SARS-CoV-2 have an efficient immune response with only mild illness and symptoms; the infection resolves within a short period [1]. However, in a subset of cases, infection causes dysfunction of both the innate and adaptive immune-response mechanisms, which results in the hyper-inflammatory state observed in severe COVID-19 patients $[2,3]$. In addition, apart from the hyper-inflammatory response, significant endothelial damage, coagulopathy, and complement hyperactivation lead to hypercoagulability and associated thromboembolism, both in terms of microangiopathy as well as in clinically evident thromboembolism [2, 4, 5].
SARS-CoV-2 infection is a disease primarily affecting the lung, and accumulating evidence shows multiple factors leading to COVID-19 pneumonia and Adult Respiratory Distress Syndrome (ARDS). First, there is direct invasion of the endothelial and lung epithelial cells of SARS-CoV-2 through high expression of angiotensin-converting enzyme 2 receptors and subsequent direct damage to the endothelial and epithelial cells. Moreover, the immune dysregulation observed in both the innate and adaptive immune mechanisms leads to cytokine over-production, hypercoagulation, complement hyperactivation, and impaired anti-inflammatory mechanisms. These mechanisms eventually lead to interstitial edema, perivascular lymphocyte, mainly T-cell, infiltration, multiple thrombi in blood vessels, and diffuse alveolar damage that is present in ARDS cases, either due to COVID-19 or unrelated. Lastly, pulmonary fibrosis can be evident at a relatively early stage of disease $[6,7]$.

Treatment can be categorized into three domains: a) administering anti-viral drugs at disease onset, b) immunomodulating and targeting the immune system's dysfunction and disrupting the hyperinflammation, and c) administering anti-thrombotic medications. Until recently, only corticosteroids appeared to reduce mortality in severe COVID-19 patients $[8,9]$, and it is the only drug that has received Surviving Sepsis Campaign (SSC) recommendation in critically ill patients [10]. Numerous studies on other immunomodulatory drugs (e.g., tocilizumab, anakinra, sarilumab) have yielded promising results. Research on IL-6 receptor antagonists has been based on the apparently important role of IL-6 receptor in the pathogenesis of COVID-19 [3] with elevated IL-6 levels predicting mortality [11]. Tocilizumab has been the most investigated and, despite initial skepticism and conflicting results, has finally demonstrated efficacy through two large randomized trials, REMAP-CAP [12] and RECOVERY trial [9]. The recently published meta-analysis by the WHO RE- 
ACT Working Group [13] has confirmed the above findings and led to the very recent addition in the WHO COVID-19 guidelines of a STRONG recommendation on "treatment with IL-6 receptor blockers (i.e., tocilizumab or sarilumab) for patients with severe or critical COVID-19 infection." However, one must still ask why it took over a year to reach this conclusion when the hypothesis was generated at the beginning of the pandemic [14]. The answer is complex, and proving the hypothesis required generation of clinical-trial data. Several trials were designed and conducted under tremendous pressure, both in terms of workload (with health-care systems overwhelmed all over the world due to the pandemic) and in terms of time with answers urgently needed. Initial randomized controlled trials failed to show the benefit of tocilizumab in reducing mortality of COVID-19 patients $[15,16]$, and this was the main reason for the drug not receiving $\mathrm{WHO}$ or SCC recommendation. There are several reasons for these inconsistent results. One main confounder was the patient-selection process. IL-6 receptor antagonists are immunomodulating drugs that inhibit hyperinflammation and immunodysregulation. But hyperinflammation must be present for these drugs to show benefit, and hospitalized COVID-19 patients without hyperinflammation, obviously, will not benefit from this treatment [17]. Hyperinflammation, characterized by molecules such as TNF $\alpha$, and IL-8, is difficult to detect in daily clinical practice [18]. However, simple clinical and laboratory tools are now available to detect hyper-inflammatory syndrome $[19,20]$ and improve patient selection. Also, most patients with hypoxemia benefit from dexamethasone, which lowered mortality and mechanical-ventilation rates. Therefore these grossly underpowered studies assessing efficacy of tocilizumab in hospitalized patients often had negative results [21]. Finally, appropriate administration timing appears to play a significant role; both REMAP-CAP and data from STOP - COVID trial showed tocilizumab to be most effective when administrated early $(<48$ hours) in patients admitted to the intensive care unit (ICU) [12, 22].

Tocilizumab is not a panacea for COVID-19 infection because hyperinflammation is neither the only pathogenetic mechanism in this disease nor the single cause of mortality [23]. Other causes, including secondary infections and thromboembolism, also affect mortality of critically ill patients. The prevalence of bacterial infections is low in hospitalized patients [24]; significantly, however, benefit of tocilizumab in intubated ICU patients may be attenuated due to the risk of increasing secondary infections [25]. In addition, thromboembolism is also an important factor in clinical deterioration and mortality for COVID-19 patients [26], and anticoagulation is far more important in such cases rather than immunomodulating treatment.

In conclusion, the recently published REMAP-CAP and RECOVERY trials, along with latest meta-analysis and the new strong recommendation by the WHO, have clarified the effectiveness of tocilizumab in critically ill COVID-19 patients confirming the molecular-pathophysiological hypothesis as well as the clinical observation of several clinicians. The success of tocilizumab has proved once again that randomized controlled trials (RCTs) are an excellent tool in medical research but only in the context of the right question and the correct design methodology. RCT results require critical reading before being accepted as established knowledge.

\section{References}

1. Gautret P, Million M, Pierre-André J, et al. Natural history of COVID-19 and therapeutic options. Expert Rev Clin Immunol. 2020; 16(12): 1159-1184.

2. Perico L, Benigni A, Casiraghi F, et al. Immunity, endothelial injury and complement-induced coagulopathy in COVID-19. Nat Rev Nephrol. 2021; 17(1): 46-64.

3. Giamarellos-Bourboulis EJ, Netea MG, Rovina N, et al. Complex Immune Dysregulation in COVID-19 Patients with Severe Respiratory Failure. Cell Host Microbe. 2020; 27(6): 992-1000.e3.

4. Evans PC, Ed Rainger G, Mason JC, et al. Endothelial dysfunction in COVID-19: a position paper of the ESC Working Group for Atherosclerosis and Vascular Biology, and the ESC Council of Basic Cardiovascular Science. Cardiovasc Res. 2020; 116(14): 2177-2184.

5. Menezes MCS, et al. SARS-CoV-2 pneumonia-receptor binding and lung immunopathology: a narrative review. Crit Care. 2021; 25(1): p. 53.

6. Vasarmidi E. Pulmonary fibrosis in the aftermath of the COVID-19 era (Review). Exp Ther Med. 2020; 20(3): 2557-2560.

7. George PM, AU Wells, RG Jenkins. Pulmonary fibrosis and COVID-19: the potential role for antifibrotic therapy. Lancet Respir Med. 2020; 8(8): 807-815.

8. Horby P. Dexamethasone in Hospitalized Patients with Covid-19. N Engl J Med. 2021; 384(8): 693-704.

9. RECOVERY Collaborative Group.Tocilizumab in patients admitted to hospital with COVID-19 (RECOVERY): a randomised, controlled, open-label, platform trial. Lancet. 2021; 397(10285): $1637-1645$.

10. Alhazzani W, et al. Surviving Sepsis Campaign Guidelines on the Management of Adults With Coronavirus Disease 2019 (COVID-19) in the ICU: First Update. Crit Care Med. 2021; 49(3): e219-e234.

11. Saridaki M. Integration of heparin-binding protein and interleukin-6 in the early prediction of respiratory failure and mortality in pneumonia by SARS-CoV-2 (COVID-19). Eur J Clin Microbiol Infect Dis. 2021; 40(7): 1405-1412.

12. Gordon AC, et al., Interleukin-6 Receptor Antagonists in Critically Ill Patients with Covid-19. N Engl J Med. 2021; 384(16): 14911502 .

13. Shankar-Hari M. Association Between Administration of IL-6 Antagonists and Mortality Among Patients Hospitalized for COVID-19: A Meta-analysis. Jama. 2021.

14. Mehta, P., et al., COVID-19: consider cytokine storm syndromes and immunosuppression. Lancet, 2020. 395(10229): p. 1033-1034.

15. Rosas IO, et al., Tocilizumab in Hospitalized Patients with Severe Covid-19 Pneumonia. N Engl J Med. 2021; 384(16): 1503-1516.

16. Stone JH, Frigault MJ, Serling-Boyd NJ, et al. Efficacy of Tocilizumab in Patients Hospitalized with Covid-19. N Engl J Med. 2020; 383(24): 2333-2344. 
17. Andrianopoulos I, Papathanasiou A, Papathanakos G, et al., Tocilizumab's efficacy in patients with Coronavirus Disease 2019 (COVID-19) is determined by the presence of cytokine storm. J Med Virol. 2021; 93(1): 120-121.

18. Valle DMD, Kim-Schulze S, Hsin-Hui H,et al. An inflammatory cytokine signature predicts COVID-19 severity and survival. Nat Med. 2020; 26(10): 1636-1643.

19. Webb BJ, Peltan ID, Jensen P, et al. Clinical criteria for COVID-19-associated hyperinflammatory syndrome: a cohort study. Lancet Rheumatol. 2020; 2(12): e754-e763.

20. Caricchio R, Gallucci M, Dass C, et al. Preliminary predictive criteria for COVID-19 cytokine storm. Ann Rheum Dis. 2021; 80(1): 88-95.

21. Huang E, Jordan SC. Jordan, Tocilizumab for Covid-19 - The Ongoing Search for Effective Therapies. N Engl J Med. 2020; 383(24): 2387-2388.

22. Gupta S, Wang W, S Hayek SS, et al. Association Between Early Treatment With Tocilizumab and Mortality Among Critically Ill Patients With COVID-19. JAMA Intern Med. 2021; 181(1): 4151.
23. Osuchowski MF, Winkler MS, Skirecki T, et al. The COVID-19 puzzle: deciphering pathophysiology and phenotypes of a new disease entity. Lancet Respir Med. 2021; 9(6): 622-642.

24. Langford BJ, So M, Raybardhan S, et al., Bacterial co-infection and secondary infection in patients with COVID-19: a living rapid review and meta-analysis. Clin Microbiol Infect. 2020; 26(12): 1622-1629.

25. Kimmig LM, Wu D, Gold M, et al. IL-6 Inhibition in Critically Ill COVID-19 Patients Is Associated With Increased Secondary Infections. Front Med (Lausanne). 2020; 7: 583897.

26. Malas MB, Naazie IN, Elsayed N, et al. Thromboembolism risk of COVID-19 is high and associated with a higher risk of mortality: A systematic review and meta-analysis. EClinicalMedicine. 2020; 29: 100639 .

To cite this article: Andrianopoulos I, Kantas D, Bajwa IA, et al. Tocilizumab's Efficacy in Severe COVID-19 Patients: Fact or Fiction. European Journal of Respiratory Medicine. 2021; 3:2.

(C) 2021 Andrianopoulos I, et al. 